\title{
Upregulation of DAPK contributes to homocysteine-induced endothelial apoptosis via the modulation of Bcl2/Bax and activation of caspase 3
}

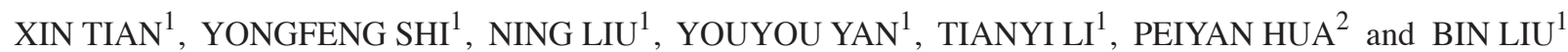 \\ Departments of ${ }^{1}$ Cardiology and ${ }^{2}$ Thoracic Surgery, The Second Hospital of Jilin University, \\ Changchun, Jilin 130041, P.R. China
}

Received August 19, 2015; Accepted August 19, 2016

DOI: $10.3892 / \mathrm{mmr} .2016 .5733$

\begin{abstract}
Hyperhomocysteinemia is characterized by an abnormally high level of homocysteine (Hcy) in the blood and is associated with cardiovascular diseases such as atherosclerosis. Endothelial dysfunction may lead to the pro-atherogenic effects associated with hyperhomocysteinemia. Endothelial dysfunction induced by Hcy has been previously investigated; however, the underlying molecular mechanism remains to be fully elucidated. The present study investigated whether death-associated protein kinase (DAPK) is involved in Hcy-induced apoptosis in human umbilical vein endothelial cells (HUVECs). It was determined that Hcy treatment upregulated the mRNA and protein expression levels of DAPK in HUVECs. Additionally, it was identified that the knockdown of DAPK using small interfering RNA may attenuate the Hcy-induced apoptosis and dissipation of mitochondrial membrane potential. DAPK inhibition may also reverse the effect of Hcy by the upregulation of B cell leukemia/lymphoma $2(\mathrm{Bcl} 2)$ and poly ADP-ribose polymerase, and the downregulation of $\mathrm{Bcl} 2$-associated $\mathrm{X}$ protein (Bax) and of caspase 3. In conclusion, the present study demonstrated that DAPK contributed to the Hcy-induced endothelial apoptosis via modulation of $\mathrm{Bcl} 2 / \mathrm{Bax}$ expression levels and activation of caspase 3 .
\end{abstract}

\section{Introduction}

Hyperhomocysteinemia, defined as elevated levels of homocysteine (Hcy) in blood plasma total is identified as an independent risk factor for the development of atherosclerosis (1-3). Previous studies have determined that the elevation of Hcy levels in the blood plasma may lead to endothelial dysfunction $(4,5)$, which

Correspondence to: Professor Bin Liu, Department of Cardiology, The Second Hospital of Jilin University, 218 Ziqiang Street, Changchun, Jilin 130041, P.R. China

E-mail: guxin33@126.com

Key words: DAPK, homocysteine, endothelial cells, apoptosis is identified as an early event in the pathogenesis of atherosclerosis $(6,7)$. A previous study reported that the apoptosis of endothelial cells contributed to endothelial dysfunction and destabilization of atherosclerotic plaques and thrombosis (8). Apoptosis or programmed cell death differs from necrosis in that it is an active process of cell suicide. In the vasculature, misdirected control of apoptosis in endothelial cells may lead to pathological conditions including inflammation, clotting and recruitment of smooth muscle cells. A previous study demonstrated that Hcy may activate a mitochondrial pathway, which may lead to reduction of the mitochondrial membrane potential $(\Delta \psi \mathrm{m})$ and induce the apoptosis of endothelial cells and result in cardiac dysfunction in vitro (9).

Death-associated protein kinase (DAPK), is an established mediator of programmed cell death (10). It has previously been observed to upregulate expression levels in atherosclerotic lesions (11). The upregulation of DAPK was observed to increase cell turnover and arterial wall instability, which increased susceptibility to low-density lipoprotein absorption (12). DAPK may function as a tumor suppressor due to its ability to promote apoptosis and autophagy $(13,14)$, suppress cellular transformation (15) and inhibit metastasis $(16,17)$. Additionally, DAPK may be activated by various stimuli, including tumor necrosis factor (TNF- $\alpha$ ), interferon- $\gamma$ and p53; therefore, acts as a converging point for apoptotic signaling (17-19). DAPK is upstream of the caspases, with the exception of caspase 8, and may induce caspase-independent cell death or autophagy. Previous studies have determined that DAPK contributed to shear stress-induced endothelial apoptosis $(20,21)$. However, the importance of DAPK in Hcy-induced apoptosis in endothelial cells remains to be fully elucidated.

The present study investigated the function of DAPK in Hcy-induced apoptosis in endothelial cells. It was determined that DAPK may contribute to the modulation of the mitochondrial pathway in Hcy-induced apoptosis in human umbilical vein endothelial cells (HUVECs).

\section{Materials and methods}

Materials. D,L-Hcy, 3-(4,5-dimethylthiazol-2-yl)-2,5-diphenyltetrazolium bromide (MTT) and rhodamine 123 (Rh123), 
a mitochondrial-specific fluorescent dye, were purchased from Sigma-Aldrich, Merck Millipore (Darmstadt, Germany). Dulbecco's modified Eagle's medium (DMEM) and fetal bovine serum (FBS) were purchased from Gibco; Thermo Fisher Scientific, Inc. (Waltham, MA, USA). Dimethyl sulfoxide (DMSO) was purchased from Shenggong Biology Engineering Technology Service, Ltd. (Shanghai, China). The Bicinchoninic Acid (BCA) protein assay kit and Annexin V-fluorescein isothiocyanate (FITC) Apoptosis Detection kit were purchased from KeyGen Biotech Co., Ltd. (Nanjing, China). Polyclonal antibodies against $\beta$-actin (cat. no. AA128; 1:1,000) and horseradish peroxidase-conjugated secondary antibodies (goat-anti rabbit; cat. no. A0208; 1:1,000; goat-anti mouse; cat. no. A0216; 1:2,000) were purchased from Beyotime Institute of Biotechnology (Shanghai, China). The DAPK antibody (cat. no. ab109382; 1:1,000) was obtained from Abcam (Cambridge, UK). Polyclonal antibodies against B cell leukemia/lymphoma 2 (Bcl2; cat. no. 2876; 1:1,000), Bcl2-associated X protein (Bax; cat. no. 2772; 1:1,000), caspase 3 (cat. no. 9662s; 1:1,000) and polyclonal ADP-ribose polymerase (PARP; cat. no. 9542; 1:1,000) were purchased from Cell Signaling Technology, Inc. (Danvers, MA, USA). The western blotting detection kit was purchased from EMD Millipore (Billerica, MA, USA).

Cell culture. HUVECs were obtained from China Center for Type Culture Collection (Wuhan, China) and maintained in DMEM supplemented with $10 \% \mathrm{FBS}$ at $37^{\circ} \mathrm{C}$ in a humidified atmosphere with $5 \% \mathrm{CO}_{2}$. The medium was changed every 2-3 days. Endothelial cells of passage 4-6 in the actively growing condition were used for the subsequent experiments.

Cell viability assay. Cell viability was evaluated by MTT assays as previously described (22). Briefly, non-transfection HUVECs were seeded in 96-well plates $\left(1.0 \times 10^{4}\right.$ cells/well $)$ and were treated with Hcy at concentrations of $0,0.1,1,5,10$ and $15 \mathrm{mM}$. After $24 \mathrm{~h}, 500 \mu \mathrm{g} / \mathrm{ml}$ MTT reagent was added and the cells were incubated for an additional $4 \mathrm{~h}$. Subsequently, $150 \mu 1$ DMSO was added to dissolve the formazan crystals. The absorbance was determined using a microplate reader (Thermo Fisher Scientific, Inc.) at $570 \mathrm{~nm}$.

Cell transfection. DAPK small interfering RNA (siRNA) and control siRNA were obtained from GenePharma Co., Ltd. (Shanghai, China). HUVECs were cultured in 6-well plates at a density of $2.0 \times 10^{5}$ cells/well for $24 \mathrm{~h}$ and then transfected with DAPK siRNA (30 $\mathrm{nM})$ or control siRNA (30 $\mathrm{nM})$ in DMEM medium without FBS using Lipofectamine 2000 transfection reagent (Invitrogen; Thermo Fisher Scientific, Inc.) according to the manufacturer's protocol.

Hoechst 33342 staining for nuclei fragmentation. HUVECs were treated with $0,5,10 \mathrm{mM}$ Hcy for $24 \mathrm{~h}$. The cells were fixed with $4 \%$ paraformaldehyde for $30 \mathrm{~min}$ at room temperature, then stained with Hoechst $33342(10 \mu \mathrm{g} / \mathrm{ml})$ at $37^{\circ} \mathrm{C}$ for $20 \mathrm{~min}$, followed by two washes with phosphate-buffered saline (PBS). Subsequently, the cells were resuspended with PBS in order to determine alterations in their nuclear morphology under a fluorescence microscope (1X71; Olympus Corporation, Tokyo, Japan).
Apoptosis analysis by flow cytometry. Flow cytometry was used to detect the apoptotic rate of HUVECs using the Annexin V-FITC/ propidium iodide (PI) staining kit. Following exposure to different concentrations of Hcy (0, 5 and $10 \mathrm{mM})$, cells were collected and washed with PBS and resuspended in binding buffer containing Annexin V-FITC and PI according to the manufacturer's protocol. Subsequent to staining, cells were analyzed using a flow cytometer.

Flow cytometry determination of $\Delta \psi m$. The alterations in $\Delta \psi \mathrm{m}$ were investigated using Rh123 and flow cytometry. Cells were seeded in 6 -well plates $\left(2.0 \times 10^{5}\right.$ cells/well $)$, transfected with siRNA, which downregulated DAPK expression for $48 \mathrm{~h}$ and then treated with $10 \mathrm{mM}$ Hcy for an additional $24 \mathrm{~h}$. Subsequently, cells were incubated with the Rh123 $(10 \mu \mathrm{M})$ at $37^{\circ} \mathrm{C}$ in the dark for $20 \mathrm{~min}$. Following filtration through 200-mesh sieve with pore size of $75 \mu \mathrm{m}$, the samples were analyzed using a flow cytometer.

Reverse transcription-quantitative polymerase chain reaction $(R T$-qPCR). Cells were collected to isolate total RNA and remove genomic DNA using TRIzol reagent (Invitrogen; Thermo Fisher Scientific, Inc.) in a sterile, RNase-free environment. Reverse transcription was performed using RevertAid Reverse Transcriptase (Thermo Fisher Scientific, Inc.) at $37^{\circ} \mathrm{C}$ for $1 \mathrm{~h}$. The qPCR reaction was performed using Maxima SYBR Green PCR Master mix (Thermo Fisher Scientific, Inc.). The $25 \mu \mathrm{l}$ final reaction volume was comprised of $12.5 \mu \mathrm{l}$ Maxima SYBR Green PCR Master mix, $0.3 \mu \mathrm{M}$ primer and $1 \mu \mathrm{l}$ cDNA. The thermocycling conditions were as follows: Initial step of $50^{\circ} \mathrm{C}$ for $2 \mathrm{~min}$, followed by a second step at $95^{\circ} \mathrm{C}$ for $15 \mathrm{~min}$, then 40 cycles of $95^{\circ} \mathrm{C}$ for $15 \mathrm{sec}$ and $60^{\circ} \mathrm{C}$ for $60 \mathrm{sec}$. Fold changes in target gene expression between treatments and controls were determined using the $2^{-\Delta \Delta C q}$ method (23), normalizing to GAPDH RNA expression as an internal reference. All results were repeated in six independent experiments and performed in triplicate each time. The following primers for qPCR were used: DAPK forward, ACA CATTGCCCTTCATCTGG and reverse AGTATTGCCGTG CCTGTCTT; GAPDH forward CGCTCTCTGCTCCTCCTG TTC and reverse ATCCGTTGACTCCGACCTTCAC.

Protein analysis. For protein analysis, cells were collected following each experiment and lysed with radioimmunoprecipitation assay buffer and ultrasound on ice. The supernatant fluids were collected following centrifugation at 13,000 x $g$ for $5 \mathrm{~min}$ at $4^{\circ} \mathrm{C}$. BCA Protein Assay kit was used to determine the protein concentrations. Gel electrophoresis was performed using $10 \%$ sodium dodecyl sulphate-polyacrylamide gel and transferred to $0.45 \mu \mathrm{m}$ polyvinylidene fluoride membranes (GE Healthcare Life Sciences, Chalfont, UK). The membranes were immersed in 5\% non-fat milk blocking buffer for $1 \mathrm{~h}$. The membranes were incubated overnight at $4^{\circ} \mathrm{C}$ with the primary antibodies $(1: 1,000)$, followed by appropriate horseradish peroxidase (HRP)-conjugated secondary antibodies $(1: 1,000)$ at room temperature for $1 \mathrm{~h}$ and Immobilon Western Chemiluminescent HRP substrate (EMD Millipore). Gel-Pro Analyzer version 6.0 (Media Cybernetics, Inc., Rockville, MD, USA) was used to extract quantitative information from the electrophoretic gels. 
A

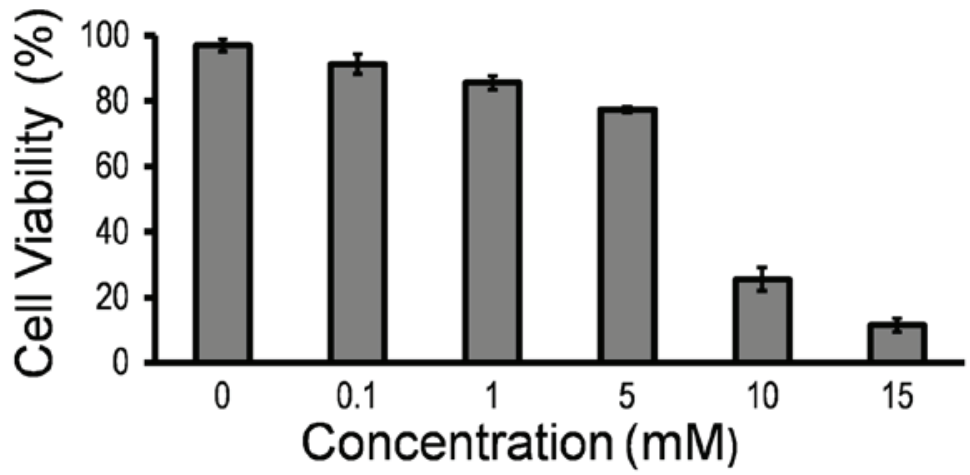

B

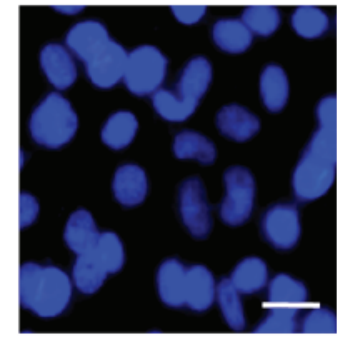

Control

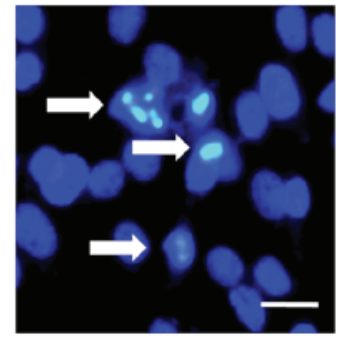

$5 \mathrm{mM}$

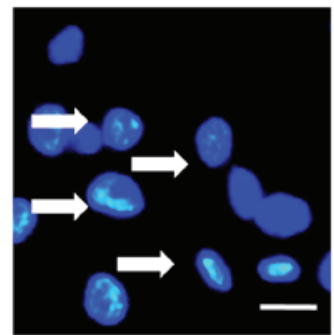

$10 \mathrm{mM}$
C

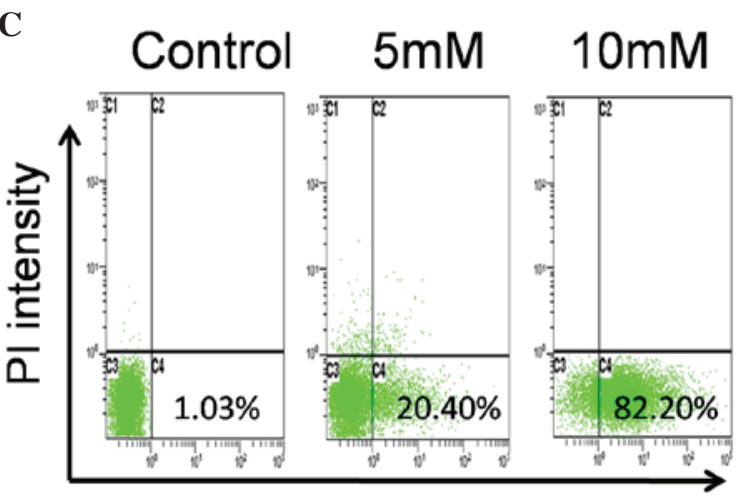

Annexin V-FITC intensity

D

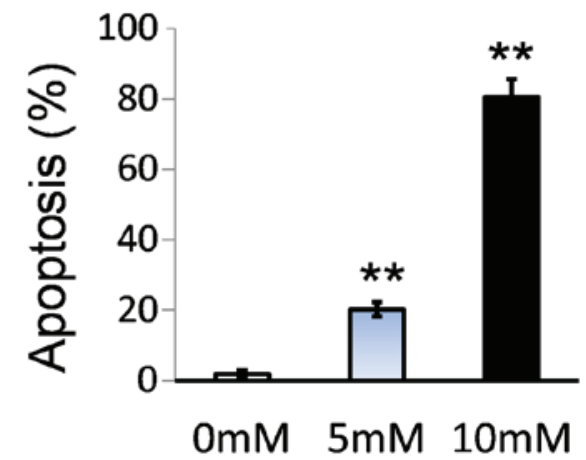

Figure 1. Hcy inhibited the growth and induced the apoptosis of HUVECs. (A) Hcy reduced the viability of HUVECs with increased concentration. (B) HUVECs exhibited apoptotic changes following exposure to Hcy, including condensed chromatin and fragmented nuclei, indicated by arrows. Scale bar, $50 \mu \mathrm{m}$. (C) Apoptosis was induced by Hcy in HUVECs as determined by flow cytometric analysis, with the quantification presented in (D). Data are expressed as the mean \pm standard deviation of three independent experiments. ${ }^{* *} \mathrm{P}<0.001$ vs. the control. Hcy, homocysteine; HUVECs, human umbilical vein endothelial cells; PI, propidium iodide; FITC, fluorescein isothiocyanate.

Statistical analysis. Data are expressed as the mean \pm standard deviation from at least three different experiments. Comparisons between groups were performed using one-way analysis of variance followed by Dunnett's test using using SPSS version 13.0 (SPSS, Inc., Chicago, IL, USA). P<0.05 was considered to indicate a statistically significant difference.

\section{Results}

Hcy reduces the survival of HUVECs and induces apoptosis. To examine the effect of Hcy on the viability of endothelial cells, HUVECs were treated with various concentrations (0.1-15 mM) of Hcy. No significant difference was identified between $0.1 \mathrm{mM}$ Hcy and the control group ( $\mathrm{P}>0.05$; Fig. 1A). Hcy at 1, 5, 10 and $15 \mathrm{mM}$ significantly inhibited cell viability in a dose-dependent manner compared with the control group $(\mathrm{P}<0.05$; Fig. 1A).

The major features of apoptotic cell death are DNA fragmentation and loss of the asymmetry of the plasma membrane. To test the effect of Hcy on cell viability, morphological changes in the nuclei were observed using Hoechst 33342 staining and fluorescence microscopy. As presented in Fig. 1B, Hcy treatment induced nuclear morphological alterations in the HUVECs, including nuclear shrinkage and DNA fragmentation. Apoptosis induced by Hcy was additionally confirmed by Annexin V-FITC/PI staining. HUVECs were treated with Hcy for $24 \mathrm{~h}$ and the percentages of cells undergoing apoptosis/necrosis were determined by flow cytometry. It was identified that Hcy may induce cell apoptosis in a dose-dependent manner (Fig. 1C). A significant increase in early apoptosis of the Hcy $5 \mathrm{mM}$ and $10 \mathrm{mM}$ treatment groups 
A

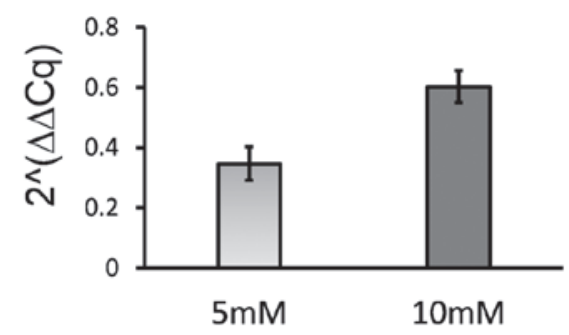

B

\section{$0 \mathrm{mM} 5 \mathrm{mM} 10 \mathrm{mM}$}

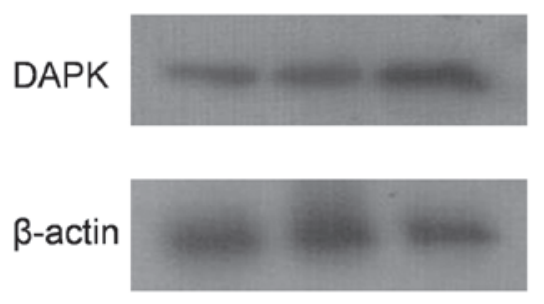

C

\section{Control siCtrl siDAPK}

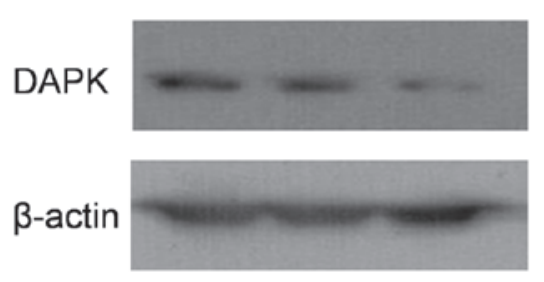

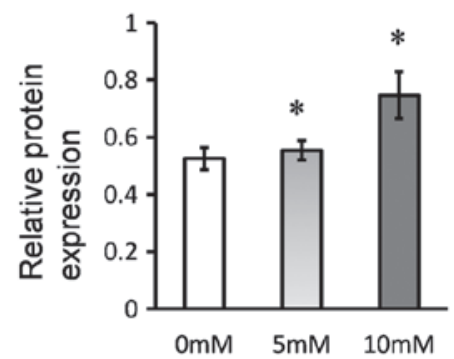

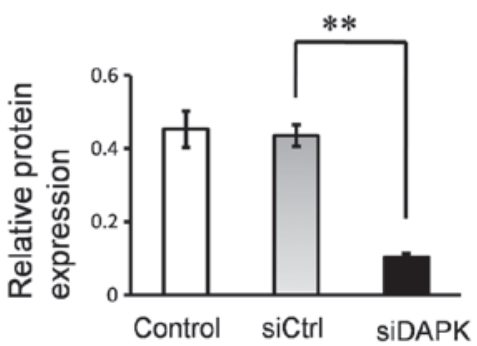

Figure 2. DAPK expression levels in HUVECs following exposure to Hcy and knockdown of DAPK. (A) DAPK mRNA expression levels in HUVECs following exposure to different concentrations of Hcy. (B) Western blot analysis of the DAPK protein expression levels in Hcy-treated HUVECs. "P<0.05 vs. ctrl. (C) Cells were transfected with siCtrl or DAPK-specific siRNA. DAPK knockdown efficiency was determined $48 \mathrm{~h}$ subsequent to transfection using western blotting. Data are expressed as the mean \pm standard deviation of three independent experiments. ${ }^{* *} \mathrm{P}<0.001$ vs. siCtrl. DAPK, death-associated protein kinase; HUVECs, human umbilical vein endothelial cells; Hcy, homocysteine; ctrl, control; siRNA, small interfering RNA.

was observed compared with the control group $(\mathrm{P}<0.001$; Fig. 1D).

Hcy increases DAPK expression in HUVECs. Following treatment with Hcy, the mRNA and protein expression levels of DAPK were determined using RT-qPCR and western blotting. It was identified that the mRNA and protein levels of DAPK were significantly upregulated in response to the $5 \mathrm{mM}$ and $10 \mathrm{mM}$ Hcy treatment groups, when compared with the control $(\mathrm{P}<0.05$; Fig. $2 \mathrm{~A}$ and $\mathrm{B})$. Hcy treatment increased DAPK mRNA and protein expression levels in HUVECs.

DAPK knockdown reduced the Hcy-induced apoptosis. To determine the effect of DAPK expression on Hcy-induced apoptosis, DAPK expression was inhibited using siRNA. DAPK expression was significantly suppressed in HUVECs compared with the non-specific control siRNA group $(\mathrm{P}<0.001$; Fig. $2 \mathrm{C})$. Following treatment with Hcy, the apoptotic rate of DAPK siRNA-transfected HUVECs was significantly reduced from 88.59 to $51.40 \%$, compared with the control group (transfected with non-specific siRNA) (P<0.05; Fig. 3A and B). These findings provide additional evidence that DAPK is important for the mediation of endothelial apoptosis induced by Hcy. Additionally, cell viability was significantly greater in the siDAPK group compared with the $10 \mathrm{mM}$ Hcy group $(\mathrm{P}<0.001$; Fig. $3 \mathrm{C})$

DAPK knockdown attenuates the effect of Hcy on $\Delta \psi m$. Hcy endothelial cells was associated with DAPK. In order to determine whether reduction of DAPK expression levels may alleviate Hcy-induced mitochondrial dysfunction, DAPK siRNA-transfected cells were treated with Hcy and the $\Delta \psi \mathrm{m}$ was evaluated. Following treatment with Hcy, an increase of $\Delta \psi \mathrm{m}$ was observed in the DAPK knockdown group from 22.98 to $53.12 \%$ (Fig. 3D). This indicated that DAPK was associated with the Hcy-induced mitochondrial dysfunction.

Knockdown of DAPK attenuates the effect of Hcy on the expression levels of apoptosis regulators. The data indicated that DAPK was involved in Hcy-induced apoptosis and with $\Delta \psi \mathrm{m}$ disruption in HUVECs. The Bcl2 protein family, a large family of apoptosis regulating proteins, modulated the mitochondrial pathway investigated in the present study. In order to characterize the function of DAPK in Hcy-induced apoptosis, the impact of reduced DAPK expression levels via siRNA transfection on Bcl2 family proteins was investigated 
A

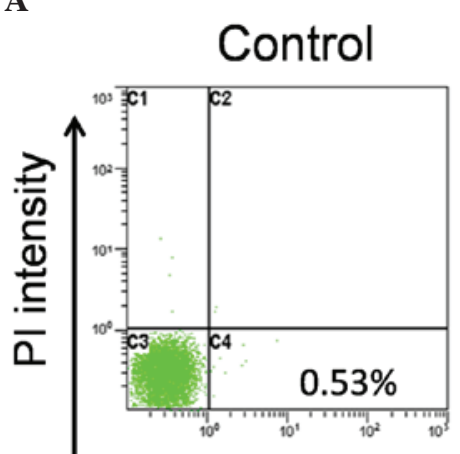

$10 \mathrm{mM}$

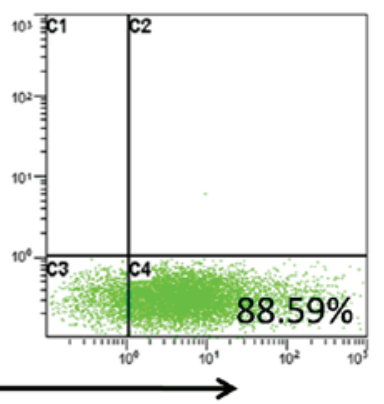

SiDAPK+

$10 \mathrm{mM}$

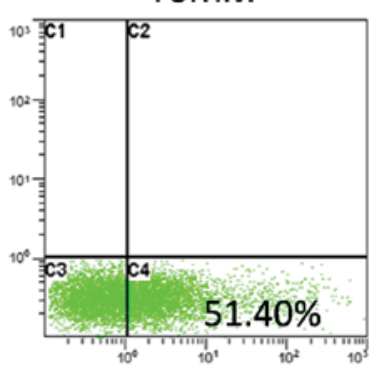

Annexin V-FITC intensity

B

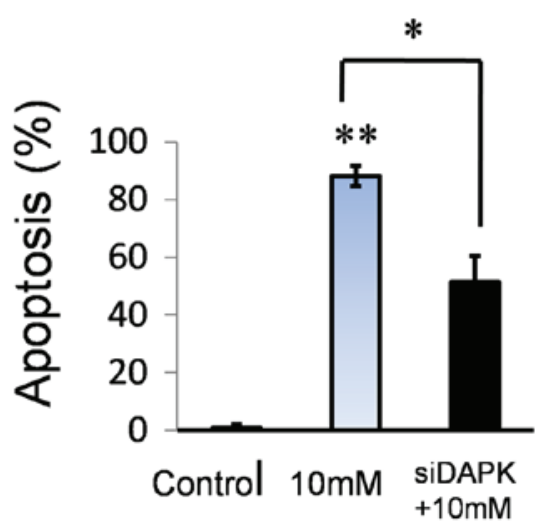

C

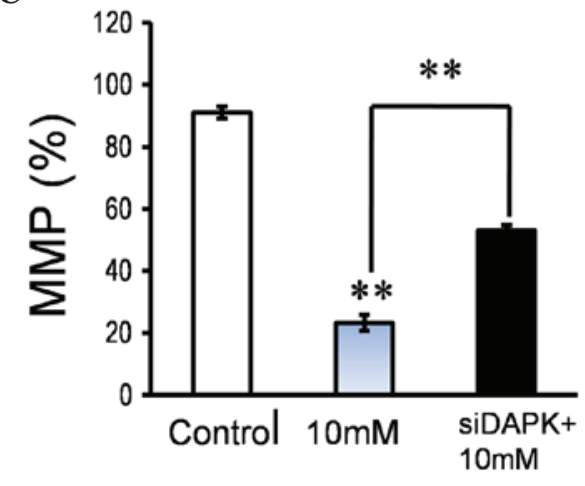

D

Control

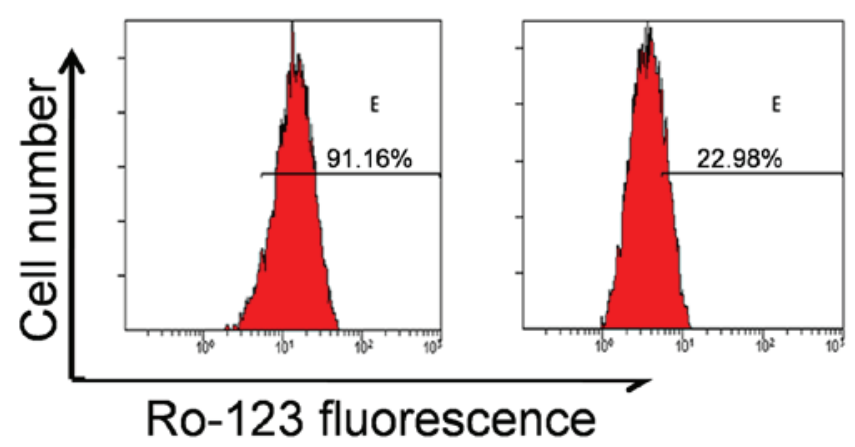

siDAPK+10mM

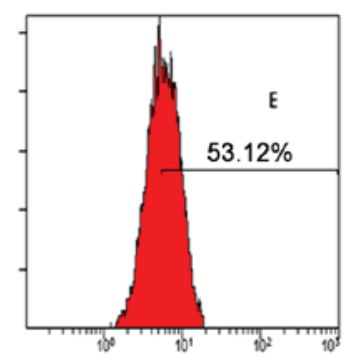

Figure 3. DAPK is required for a Hcy-induced apoptosis and $\Delta \psi \mathrm{m}$ change. (A) Transfection with siDAPK reduced the apoptosis induced by Hcy. Quantification of the (B) apoptotic rate and (C) $\Delta \psi \mathrm{m}$ following siDAPK transfection. Data are expressed as the mean \pm standard deviation of three independent experiments. ${ }^{*} \mathrm{P}<0.05$ and ${ }^{* *} \mathrm{P}<0.001$ vs. control. (D) Flow cytometric analysis. The values indicate the percentages of rhodamine 123 fluorescence on HUVECs subsequent to treatment. Data shown are representative of three independent experiments. DAPK, death-associated protein kinase; Hcy, homocysteine; $\Delta \psi \mathrm{m}$, mitochondrial membrane potential; siDAPK, small interfering DAPK; HUVECs, human umbilical vein endothelial cells; PI, propidium iodide; FITC, fluorescein isothiocyanate.

in HUVECs treated with Hcy using western blot analysis (Fig. 4). As presented in Fig. 4A and C, Hcy treatment significantly reduced the ratio of $\mathrm{Bcl} 2$ to $\mathrm{Bax}(\mathrm{P}<0.05)$, whereas the knockdown of DAPK significantly reversed the effect of Hcy treatment on $\mathrm{Bax}$ and $\mathrm{Bcl} 2$ levels $(\mathrm{P}<0.05)$. In addition, caspase 3 and PARP activation was examined. Hcy treatment resulted in significantly increased caspase 3 cleavage and reduced PARP expression levels in HUVECs $(\mathrm{P}<0.01$; Fig. 4C). Caspase 3 cleavage, characterized by the appearance of 17 and $19 \mathrm{kDa}$ protein band and PARP cleavage characterized by a $89 \mathrm{kDa}$ protein band reduced in cells transfected with siDAPK compared with the cells transfected with non-specific siRNA. Cleaved caspase 3 expression levels were significantly reduced in the siDAPK group compared with the $10 \mathrm{mM}$ Hcy treatment group $(\mathrm{P}<0.05$; Fig. $4 \mathrm{C})$.

\section{Discussion}

The present study investigated the importance of DAPK for the possible mechanism that triggers Hcy-induced apoptosis in HUVECs. Initially, it was confirmed that Hcy reduced the viability of HUVECs and induced apoptosis. In addition, it was determined that Hcy upregulated DAPK expression in a dose-dependent manner. Additionally, it was revealed that inhibition of DAPK may attenuate apoptosis and dissipation of the $\Delta \psi \mathrm{m}$. The underlying molecular mechanisms were also 
A
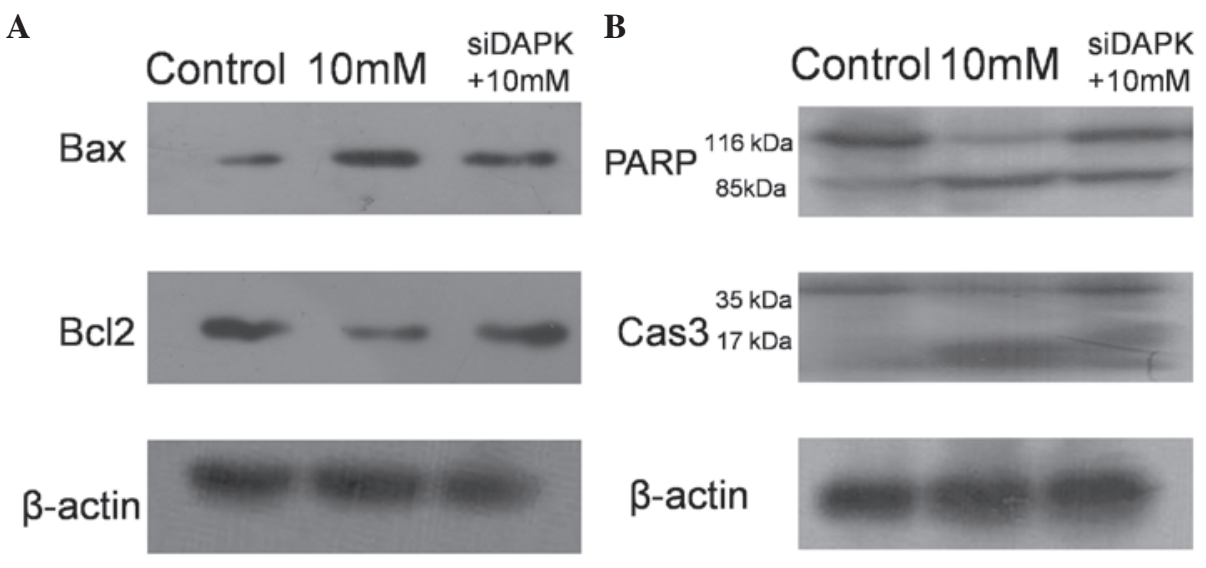

$\beta$-actin

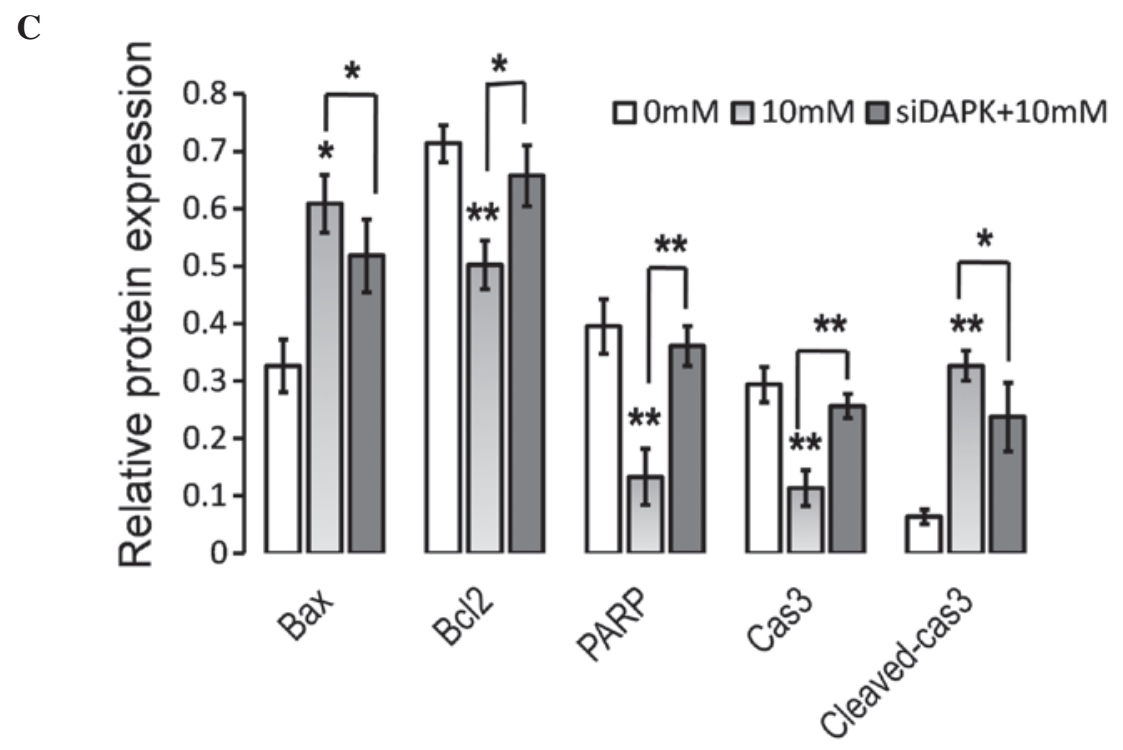

Figure 4. Suppression of DAPK attenuated the effect of Hcy on the expression levels of apoptosis regulators. HUVECs were transfected with DAPK-specific siRNA and $48 \mathrm{~h}$ subsequent to transfection, cells were treated with Hcy $(10 \mathrm{mM})$ for $24 \mathrm{~h}$. Whole-cell extracts were prepared and probed for (A) Bcl2, Bax, (B) PARP and Cas3 by western blot analysis. (C) Quantification of the western blotting. Data are expressed as the mean \pm standard deviation of three independent experiments. " $\mathrm{P}<0.05$ and ${ }^{* *} \mathrm{P}<0.01$ vs. control. DAPK, death-associated protein kinase; Hcy, homocysteine; HUVECS, human umbilical vein endothelial cells; siRNA, small interfering RNA; Bcl2, B cell leukemia/lymphoma 2; Bax, Bcl2-associated X protein; PARP, poly ADP-ribose polymerase; Cas3, caspase 3.

investigated and it was determined that DAPK participated in Hcy-induced apoptosis by reducing the ratio of Bcl2/Bax and activation of caspase 3 in HUVECs.

Increased levels of Hcy in blood plasma have been identified as an independent risk factor for the development of atherosclerosis (1-3). In atherosclerosis, increased apoptosis has been established to contribute to prolonged inflammatory response, plaque instability, rupture and thrombus formations (11). Previous studies have also reported apoptosis of endothelial cells associated with Hcy (24-27). The results of the present study are in agreement with this, as it was evident that Hcy reduced cell viability and induced apoptosis in a dose-dependent manner.

DAPK is an important protein kinase for the modulation of apoptotic pathways (28). A previous study identified that DAPK functioned as a positive mediator of apoptosis induced by various stimuli, including TNF- $\alpha$, interferon- $\gamma$ and p53 (29). It was previously reported that DAPK activity may be involved in TNF- $\alpha$-induced apoptotic pathways in bovine aortic endothelial cells (20). To the best of our knowledge, the present study is the first to reported that Hcy treatment increased mRNA and protein DAPK expression levels. This suggested that DAPK may contribute to the apoptotic effect of Hcy in endothelial cells. When inhibition of DAPK was induced, a reduction in Hcy-induced apoptosis was observed in endothelial cells, indicating that DAPK is crucial for the mediation of Hcy-induced apoptosis.

A previous study determined that $\Delta \psi \mathrm{m}$ loss, an early occurring event, may be directly associated with apoptosis (30). As $\Delta \psi \mathrm{m}$ is reduced, mitochondrial permeability transition pores are opened and in turn release cytochrome $c$ and other pro-apoptotic molecules from the intermembrane space into the cytosol. It has been previously demonstrated that Hcy activates the mitochondrial pathway leading to a reduction in $\Delta \psi \mathrm{m}$ and apoptosis in cardiac microvascular endothelial cells (26). The current study determined that Hcy treatment resulted in a reduction of $\Delta \psi \mathrm{m}$ in HUVECs, which is consistent with previous studies $(26,31)$. It is of note, that the reduction of $\Delta \psi \mathrm{m}$ may be attenuated by the knockdown of DAPK, as it has been identified 
to be important for Hcy-induced mitochondrial dysfunction. Therefore, this may be the possible mechanism behind endothelial apoptosis induced by Hcy.

$\mathrm{Bcl} 2$ family proteins are involved in the mitochondria-dependent apoptosis pathway, which includes anti-apoptotic proteins and pro-apoptotic proteins such as $\mathrm{Bcl} 2$ and $\mathrm{Bax}$ (32). The present study demonstrated that following Hcy treatment the protein expression levels of Bax were increased, whereas the expression levels of $\mathrm{Bcl} 2$ were reduced. It is of note that downregulation of DAPK expression reversed this response. Caspase activation is one of the processes that signify the onset of apoptosis, and caspase 3 has been considered to be a central component of the proteolytic cascade during apoptosis, as it may cleave various nuclear proteins, including PARP, which may lead to atypical apoptotic DNA fragmentation (33). The present study demonstrated that exposure to Hcy activated caspase 3, whereas the knockdown of DAPK reduced this activation. Therefore, it is possible that Hcy-induced apoptosis in HUVECs is associated with modulation of the $\mathrm{Bcl} 2 / \mathrm{Bax}$ ratio and activation of caspase-3 by DAPK.

\section{Acknowledgements}

The present study was supported by the Wu Jieping Medical Foundation (grant no. 320.6750.12265).

\section{References}

1. Guilland JC, Favier A, Potier de Courcy G, Galan P and Hercberg S: Hyperhomocysteinemia: An independent risk factor or a simple marker of vascular disease? 1. Basic data. Pathol Biol (Paris) 51: 101-110, 2003.

2. Lawrence de Koning AB, Werstuck GH, Zhou J and Austin RC: Hyperhomocysteinemia and its role in the development of atherosclerosis. Clin Biochem 36: 431-441, 2003.

3. Clarke R, Daly L, Robinson K, Naughten E, Cahalane S, Fowler B and Graham I: Hyperhomocysteinemia: An independent risk factor for vascular disease. N Engl J Med 324: $1149-1155,1991$.

4. Horvath B, Szapary L, Debreceni L, Feher G, Kenyeres P, Fulop A, Battyani I and Toth K: Effect of Sclerovit on endothelial dysfunction, hemorheological parameters, platelet aggregation, plasma concentration of homocysteine and progression of atherosclerosis in patients with vascular diseases. Clin Hemorheol Microcirc 42: 19-28, 2009.

5. Briasoulis A, Tousoulis D, Androulakis ES, Papageorgiou N, Latsios G and Stefanadis C: Endothelial dysfunction and atherosclerosis: Focus on novel therapeutic approaches. Recent Pat Cardiovasc Drug Discov 7: 21-32, 2012.

6. Davignon J and Ganz P: Role of endothelial dysfunction in atherosclerosis. Circulation 109 (23 Suppl 1): III27-III32, 2004.

7. Martin BJ and Anderson TJ: Risk prediction in cardiovascular disease: The prognostic significance of endothelial dysfunction. Can J Cardiol 25 (Suppl A): A15-A20, 2009.

8. Chen F, Eriksson P, Kimura T, Herzfeld I and Valen G: Apoptosis and angiogenesis are induced in the unstable coronary atherosclerotic plaque. Coron Artery Dis 16: 191-197, 2005.

9. Kumar D and Jugdutt BI: Apoptosis and oxidants in the heart. J Lab Clin Med 142: 288-297, 2003.

10. Cohen O and Kimchi A: DAP-kinase: From functional gene cloning to establishment of its role in apoptosis and cancer. Cell Death Differ 8: 6-15, 2001.

11. Martinet W, Schrijvers DM, De Meyer GR, Thielemans J, Knaapen MW, Herman AG and Kockx MM: Gene expression profiling of apoptosis-related genes in human atherosclerosis: Upregulation of death-associated protein kinase. Arterioscler Thromb Vasc Biol 22: 2023-2029, 2002.
12. Schumacher AM, Velentza AV and Watterson DM: Death-associated protein kinase as a potential therapeutic target. Expert Opin Ther Targets 6: 497-506, 2002.

13. Inbal B, Bialik S, Sabanay I, Shani G and Kimchi A: DAP kinase and DRP-1 mediate membrane blebbing and the formation of autophagic vesicles during programmed cell death. J Cell Biol 157: 455-468, 2002.

14. Bialik S and Kimchi A: The death-associated protein kinases: Structure, function, and beyond. Annu Rev Biochem 75: 189-210, 2006.

15. Raveh T, Droguett G, Horwitz MS, DePinho RA and Kimchi A: DAP kinase activates a p19ARF/p53-mediated apoptotic checkpoint to suppress oncogenic transformation. Nat Cell Biol 3: 1-7, 2001.

16. Michie AM, McCaig AM, Nakagawa R and Vukovic M: Death-associated protein kinase (DAPK) and signal transduction: Regulation in cancer. FEBS J 277: 74-80, 2010.

17. Chen RH, Wang WJ and Kuo JC: The tumor suppressor DAP-kinase links cell adhesion and cytoskeleton reorganization to cell death regulation. J Biomed Sci 13: 193-199, 2006.

18. Cohen O, Inbal B, Kissil JL, Raveh T, Berissi $H$, Spivak-Kroizaman T, Feinstein E and Kimchi A: DAP-kinase participates in TNF-alpha- and Fas-induced apoptosis and its function requires the death domain. J Cell Biol 146: 141-148, 1999.

19. Pelled D, Raveh T, Riebeling C, Fridkin M, Berissi H, Futerman $\mathrm{AH}$ and Kimchi A: Death-associated protein (DAP) kinase plays a central role in ceramide-induced apoptosis in cultured hippocampal neurons. J Biol Chem 277: 1957-1961, 2002.

20. Rennier K and Ji JY: Shear stress regulates expression of death-associated protein kinase in suppressing TNF $\alpha$-induced endothelial apoptosis. J Cell Physiol 227: 2398-2411, 2012.

21. Rennier K and Ji JY: Effect of shear stress and substrate on endothelial DAPK expression, caspase activity, and apoptosis. BMC Res Notes 6: 10, 2013.

22. Hua P, Sun M, Zhang G, Zhang Y, Tian X, Li X, Cui R and Zhang $\mathrm{X}$ : Cepharanthine induces apoptosis through reactive oxygen species and mitochondrial dysfunction in human non-small-cell lung cancer cells. Biochem Biophys Res Commun 460: 136-142, 2015.

23. Livak KJ and Schmittgen TD: Analysis of relative gene expression data using real-time quantitative PCR and the 2(-Delta Delta C(T)) method. Methods 25: 402-408, 2001.

24. Suhara T, Fukuo K, Yasuda O, Tsubakimoto M, Takemura Y, Kawamoto H, Yokoi T, Mogi M, Kaimoto T and Ogihara T: Homocysteine enhances endothelial apoptosis via upregulation of Fas-mediated pathways. Hypertension 43: 1208-1213, 2004.

25. Zhang C, Cai Y, Adachi MT, Oshiro S, Aso T, Kaufman RJ and Kitajima S: Homocysteine induces programmed cell death in human vascular endothelial cells through activation of the unfolded protein response. J Biol Chem 276: 35867-35874, 2001.

26. Tyagi N, Ovechkin AV, Lominadze D, Moshal KS and Tyagi SC: Mitochondrial mechanism of microvascular endothelial cells apoptosis in hyperhomocysteinemia. J Cell Biochem 98: 1150-1162, 2006.

27. Sipkens JA, Hahn N, van den Brand CS, Meischl C, Cillessen SA, Smith DE, Juffermans LJ, Musters RJ, Roos D, Jakobs C, et al: Homocysteine-induced apoptosis in endothelial cells coincides with nuclear NOX2 and peri-nuclear NOX4 activity. Cell Biochem Biophys 67: 341-352, 2013.

28. Kimchi A: DAP kinase and DAP-3: Novel positive mediators of apoptosis. Ann Rheum Dis 58 (Suppl 1): I14-I19, 1999.

29. Yoo HJ, Byun HJ, Kim BR, Lee KH, Park SY and Rho SB: DAPk1 inhibits NF- $\mathrm{BB}$ activation through TNF- $\alpha$ and INF- $\gamma$-induced apoptosis. Cell Signal 24: 1471-1477, 2012.

30. Lemasters JJ: V. Necrapoptosis and the mitochondrial permeability transition: Shared pathways to necrosis and apoptosis. Am J Physiol 276: G1-G6, 1999.

31. Dong D, Wang B, Yin W, Ding X, Yu J and Kang YJ: Disturbance of copper homeostasis is a mechanism for homocysteine-induced vascular endothelial cell injury. PloS One 8: e76209, 2013.

32. King KL and Cidlowski JA: Cell cycle regulation and apoptosis. Annu Rev Physiol 60: 601-617, 1998.

33. Porter AG and Jänicke RU: Emerging roles of caspase-3 in apoptosis. Cell Death Differ 6: 99-104, 1999. 